\title{
Editorial
}

\section{Redaktionswechsel bei der ZSPM zum 1.2.2000}

In der Leitung der Zeitschrift für Sozial- und Präventivmedizin (ZSPM) wird es zum Ubergang in das neue Jahrtausend einen Wechsel geben. Der Chefredaktor Robert Steffen und dessen Redaktionsassistentin Janine Schiller (ISPM Zürich) treten zurück. Das Engagement von Robert Steffen für die Zeitschrift war in den letzten Jahren vor allem auf die Sehaffung tragfähiger struktureller Voraussetzungen, never nationaler und internationaler Allianzen und daraus resultierend einer ausreichenden Finanzbasis ausgerichtet. Die herausragende Neuerung diesbezúglich ist eine umfassende Partnerschaft mit der "Stiftung 19", der Schweizerischen Stiftung für Gesundheitsförderung.

Robert Steffen übergibt nach sechs Jahren zum 1. Februar 2000 die Leitung der Redaktion an Thomas Abel am ISPM Bern und Alfredo Morabia am HU Genéve (ab August 2000). Redaktionsassistentin in Bern wird Nicole Graf. Die beiden neuen Chefredaktoren werden auf der Arbeit von Robert Steffen aufbauen können, um mit der ZSPM die neuen Herausforderungen einer sich rasch wandelnden Landschaft der Public HealthForschung und -Praxis konstruktiv und erfolgreich angehen zu können. Die Schweizerische Gesellschaft für Prävention und Gesundheitswesen (SGPG) als Hauptträgerin der Zeitschrift und die neue Redaktion sind sich einig, dass die ZSPM auf diese neuen Herausforderungen mit einer Neuorientierung antworten muss. Unter der Leitung von Thomas Abel und Alfredo Morabia wird es stringentere inhaltiche Fokussierungen, neve Balancen zwischen der nationalen und der internationalen Ausrichtung sowie erweiterte formen der partnerschaftlichen Kooperationen geben. Dabei wird die ZSPM in Zukunft auf die beiden Bereiche Gesundheitsbefragungen und Gesundheitsförderung fokussieren und sich bemuhen, mit deren Verbindung zunehmend wichtigen Teil von Public Health in innovativer Weise voranzutreiben. Bel der grossen Zahl internationaler Public Health-Fachzeitschriften sollen diese Änderungen der ZSPM helfen, eine markante und gesicherte Position in diesem Markt zu entwickeln. Ein Feinkonzept zur Neugestaltung wird derzeit ausgearbeitet und in einer der kommenden Ausgaben der ZSPM ausführlicher dargestellt werden.

Die Ubergangsarbeiten werden sich in drei zeitliche Phasen gliedern. In Phase 1 (ab 1.2.2000) wird die Übergabe der Chefredaktion von Robert Steffen an Thomas Abel und die technische Leitung von Janine Schiller an Nicole Graf durchgeführt. Phase 2 (ab. 1.8.2000) wird dazu dienen, die Details der inhaltilchen und organisatorischen Neugestaltung auszuarbeiten und umzusetzen. In dieser Phase sollen dann auch personelle Umstellungen im Editorial Board durchgefuhrt werden. Die Umstrukturierungen sollen dann am 1.1.2001 abgeschlossen sein und ab dann in Phase 3 wird die neue ZSPM in neuem Gewand erscheinen.

Die abtretende Redaktionsleitung wie auch das neue Leitungsteam danken Ihnen, liebe Leserlinnen, aber auch den zahlreichen Expertinnen, für Ihr anhaltendes interesse an unserer Fachzeitschrift und hoffen, dass Sie mit uns gemeinsam die neuen Herausforderungen und Chancen einer modernen schweizerischen und europaischen Public Health annehmen werden.

Thomas Abel, Alfredo Morabia und Robert Steffen 